\title{
Prevalence of Human T-Cell Leukemia Virus Type 1 Carrier in Japanese Pregnant Women in 2013
}

\author{
Shunji Suzukia, b, Masanobu Tanaka ${ }^{a}$, Hideo Matsuda ${ }^{a}$, Yuki Tsukahara ${ }^{a}$, Yasushi Kuribayashia ${ }^{a}$ \\ Akihito Nakai ${ }^{a}$, Ryoichiro Miyazakia ${ }^{a}$ Naoki Kamiya ${ }^{a}$, Akihiko Sekizawa ${ }^{a}$, Nobuko Mizutani ${ }^{a}$, \\ Katsuyuki Kinoshita ${ }^{a}$
}

\section{To the Editor}

Since September 2010, in Japan serological screening for the detection of human T-cell leukemia virus type 1 (HTLV-1) antibodies can be performed for all women during pregnancy with the Japanese public funds for strategies for prevention of HTLV-1 vertical transmission, because Japan, especially Kyushu area, has been reported to be one of the areas of highest prevalence of HTLV-1 in the world [1, 2]. In our previous study [3], we examined the prevalence of HTLV-1 carrier in Japanese pregnant women according to the implement rate and results of HTLV-1 screening and confirmation tests of women who gave births in Japan in 2011. The total rates of positive HTLV-1 screening tests and positive western blot (WB) test in positive screening tests were $0.32 \%$ and $49.8 \%$, respectively in 2011. Considering the response rate and the rate of implementation of WB test, the number of HTLV-1 carrier in Japanese pregnant women in 2011 was estimated to be $1,560(0.15 \%)$. In addition, although the number of delivery in Kyushu area was only $14 \%$ of Japanese deliveries, $53 \%$ of HTLV- 1 carrier of Japanese pregnant women was present in Kyushu area.

Recently, the migration of Japanese people from Kyushu area to the metropolitan areas has been thought to contribute to a significant decrease of HTLV-1 carriers in Kyushu area and an increase in Kanto (including Tokyo) area in Japan [1, 4]. To confirm this migration in Japanese pregnant women, on December 2014, we requested again 2,544 obstetrical facilities that are members of Japan Association of Obstetricians and Gynecologists (JAOG) to provide information of HTLV-1 tests in pregnant women who delivered at $\geq 22$ weeks' gestation in 2013. A total of $1,356(53.3 \%)$ of 2,544 obstetrical facilities responded and information on a total of 538,167 women, accounting for approximately $54 \%$ of all deliveries that occurred in Japan during the study period (approximately 1,001,800 births) was provided.

Manuscript accepted for publication January 12, 2015

aJapan Association of Obstetricians and Gynecologists, Japan

${ }^{b}$ Corresponding Author: Shunji Suzuki, Department of Obstetrics and Gynecology, Japanese Red Cross Katsushika Maternity Hospital, 5-11-12 Tateishi, Katsushika-ku, Tokyo 124-0012, Japan. Email: czg83542@mopera.ne.jp

doi: http://dx.doi.org/10.14740/jocmr2097w
In 2013, the total rates of positive HTLV-1 screening tests and positive WB test in positive screening tests were $0.35 \%$ and $50.8 \%$, respectively. Considering the response rate and the rate of implementation of WB test, the number of HTLV-1 carrier in Japanese pregnant women in 2013 was estimated to be $1,780(0.18 \%)$. Table 1 shows the difference in the estimated number of HTLV-1 carrier based on positive WB test by area in Japan between 2011 and 2013. The estimated number of HTLV-1 carrier in 2013 seemed to be more than that in 2011, especially in the northeast and southwest (Kyushu) areas. In addition, $51 \%$ of HTLV-1 carrier of Japanese pregnant women was present in Kyushu area, although the number of delivery in Kyushu area was only 13\% of Japanese deliveries in 2013.

Although the migration of Japanese people from Kyushu area to the metropolitan areas has been supposed to contribute to a significant decrease of HTLV-1 carriers in Kyushu area, the estimated number of HTLV-1 carrier of pregnant women seemed to be increased in Kyushu area. In addition, the estimated rate of HTLV-1 carrier in pregnant women in Kyushu area was still significantly higher than that in the other areas $(\mathrm{P}<0.01$ by the Chi-square test). Therefore, there are still remaining problems concerning the locality for strategies for prevention of HTLV-1 vertical transmission in Japan.

\section{Declaration of Interest}

The authors report no conflicts of interest. The authors are responsible for the content and writing of the paper. This study was supported by the Japan Health, Labour and Welfare scientific research subsidies: "HTLV-1 mother-to-child transmission prevention research: cohort research of HTLV-1 antibodypositive pregnant women".

\section{References}

1. Watanabe T. Current status of HTLV-1 infection. Int J Hematol. 2011;94(5):430-434.

2. Mylonas I, Bruning A, Kainer F, Friese K. HTLV infection and its implication in gynaecology and obstetrics. Arch Gynecol Obstet. 2010;282(5):493-501.

3. Suzuki S, Tanaka M, Matsuda H, Tsukahara Y, Kuribayashi Y, Gomibuchi H, Miyazaki R, et al. Current status of HTLV-1 carrier in Japanese pregnant women. J Matern 
Table 1. Results of HTLV-1 Western Blot Tests and Estimated Number of Human T-Cell Leukemia Virus Type 1 Carrier in Pregnant Women Who Delivered at $\geq 22$ Weeks' Gestation in 2011 and 2013 by Area in Japan

\begin{tabular}{|c|c|c|c|c|}
\hline & \multicolumn{2}{|r|}{2011} & \multicolumn{2}{|r|}{2013} \\
\hline & WB positive rate (\%) & Estimated HTLV-1 carrier & WB positive rate $(\%)$ & Estimated HTLV-1 carrier \\
\hline Northeast area & 0.084 & 90 & 0.140 & 150 \\
\hline Central area & 0.083 & 120 & 0.080 & 110 \\
\hline Kinki (around Osaka) & 0.151 & 240 & 0.163 & 270 \\
\hline Kyushu area (southwest area) & 0.596 & 820 & 0.663 & 910 \\
\hline Total & 0.154 & 1,560 & 0.178 & 1,780 \\
\hline
\end{tabular}

Values are presented as percentage or number. HTLV-1: human T-cell leukemia virus type 1; WB test: western blot test.

Fetal Neonatal Med. 2014;27(3):312-313.

4. Satake M, Yamaguchi K, Tadokoro K. Current prevalence of HTLV-1 in Japan as determined by screening of blood donors. J Med Virol. 2012;84(2):327-335. 